\title{
Special Issue on Health and Wellbeing
}

\author{
Jean Gelissen $^{1} \cdot$ Daniel Sonntag ${ }^{2}$
}

Published online: 31 March 2015

(c) Springer-Verlag Berlin Heidelberg 2015

\begin{abstract}
This special issue of KI Journal on health and wellbeing brings together a collection of articles on selfmonitoring (quantified-self) related to health and habits (these solutions should reduce the costly demand for secondary prevention) and big health data analysis towards clinical data intelligence for individualized patient treatment. Others include invited contributions on intelligent user interfaces for health and wellbeing. The articles in this collection address diverse aspects of AI methods in the patient/user centric view, the doctor/clinical view, or the combination of these two views.
\end{abstract}

The EU28 population's annual healthcare expenditure has risen to $€ 1,085$ billion, a substantial share of which arises through secondary prevention, long-term care and homecare ( $€ 90$ billion). These costs are increasing towards 2020 , while the available budget and the number of caretakers are shrinking. In developed countries around the world, an ageing population is the new reality. It's a reality that poses challenges to society, but also unique opportunities for artificial intelligence methods in health and wellbeing.

One of the biggest challenges is the reduction of the demand for expensive healthcare by detecting small physical and mental health issues early and avoiding larger

$\triangle$ Jean Gelissen

jean.gelissen@eitictlabs.eu

Daniel Sonntag

sonntag@dfki.de

1 EIT ICT Labs, Eindhoven, Netherlands

2 DFKI, Saarbrücken, Germany health problems by suitable lifestyle interventions in the form of primary prevention. Four specific lifestyle factors (not smoking, maintaining a healthy weight, regular exercise, and following a healthy diet) together are associated with as much as an 80 percent reduction in the risk of developing the most common and deadly chronic diseases. In other words, an ounce of prevention is worth a pound of cure. Next to improving the quality of life, the reduction of the healthcare costs by primary prevention contributes to the UE 2020 goals of extending the labor force participation by 2 years and the extension of the period of independent living by also 2 years.

We should act on this challenge by offering AI-based solutions that respond to the demands for (1) selfmonitoring (quantified-self) related to health and habits, while these solutions will also reduce the costly demand for secondary prevention, as well as cure and care for caretakers and insurers; (2) big health data analysis and clinical data intelligence for individualized treatment.

The user centric view includes smart unobtrusive sensing of vital body signals (of care home residents), event/task extraction from (video) life logging, data mining of contextual data, personalised associations between health and behaviour, vital signs and context data fusion and correlation, smart coaching algorithms (life-coaching) for wellbeing, intelligent user interfaces for health and wellbeing, adaptive persuasion (based on a feedback loop) and persuasion technologies, adaptable interfaces that understand the physical and cognitive abilities of the user, adaptive interfaces that learn so that the user becomes more comfortable with the service over time, personalized schemes for behavioral change, health risk assessment for individuals and specific target groups, agents and healthcare, and last but not least, embodied conversational agents (ECAs) in healthcare. 
The clinical view is about smart (unobtrusive) sensing of vital body signals in clinical environments, data mining of contextual clinical data in different modalities (e.g., clinical records and medical images), semantic annotation of medical texts and images related to the ageing population, text mining in the health and wellbeing domain, big data analysis and clinical data intelligence, personalized schemes for individualised treatment and medication, and formalizing clinical guidelines for health and wellbeing.

In the medical informatics domain, three major tracks can be identified, namely medical information systems, data intelligence (analytics of medical data), and human factors. Several contributions in this special issue fall into the system track which focuses on healthcare systems architectures, frameworks, and applications. The data intelligence track emphasizes data processing, mining and retrieval as well as knowledge discovery. The human factors include special aspects of intelligent user interfaces such as understanding of user contexts and interface design of healthcare applications.

\section{Content}

\subsection{Technical Contributions}

- Rafal Kocielnik, Natalia Sidorova: Personalized Stress Management: Enabling Stress Monitoring with LifelogExplorer

- Anders Hedman, Josef Hallberg: Cognitive Endurance for Brain Health: Challenges of Creating an Intelligent Warning System

- Sonja Zillner, Sabrina Neururer: Technology Roadmap Development for Big Data Healthcare Applications

- Magnus Boman, Pedro Sanches: Sensemaking in Intelligent Health Data Analytics

- Denis Krompaß, Cristóbal Esteban, Volker Tresp, Martin Sedlmayr, Thomas Ganslandt: Exploiting Latent Embeddings of Nominal Clinical Data for Predicting Hospital Readmission

- Christine Lisetti: Now all together: Overview of Virtual Health Assistants Emulating Face-to-Face Health Interview Experience

\subsection{Research Projects}

- Marc Cavazza, Fred Charles, Alan Lindsay, Jonathan Siddle, Gersende Georg: An Interactive Narrative Format for Clinical Guidelines

\subsection{Interviews}

- Jörg Habetha: A Paradigm Shift in Healthcare Provision

\subsection{AI Market}

- Richard McClatchey: Facilitating Evolution during Design and Implementation

\subsection{Discussions}

- András Lörincz: Revolution in Health and WellbeingMachine learning, crowdsourcing and self-annotation

\section{Service}

\subsection{Major Projects and Organizations}

- World Health Organization (WHO): http://www.who. int

- World Health Organization Europe: http://www.euro. who.int

- EIT-ICT Labs on H\&W (Horizon 2020): http://www. eitictlabs.eu/innovation-entrepreneurship/healthwellbeing/

- Clinical Data Intelligence (BMWi): http://www.klinischedatenintelligenz.de

- Medical Cyber-Physical Systems

- Workshop: http://www.workshop.medcps.org

- EIT Project: http://www.dfki.de/MedicalCPS/

- Philips Healthcare: http://www.medical.philips.com

- Siemens Healthcare: http://www.healthcare.siemens. com

- WatsonPaths (IBM): http://www.research.ibm.com/cog nitive-computing/watson/watsonpaths.shtml

- Cerner healthcare systems: http://www.cerner.com

- McKesson health care systems: http://www.mckesson. com

- National Center for Biotechnology Information: http:// www.ncbi.nlm.nih.gov

- HITECH Act: http://www.en.wikipedia.org/wiki/Health_ Information_Technology_for_Economic_and_Clinical_ Health_Act

- RAND Health and Well-Being in the Home: http:// www.rand.org/content/dam/rand/pubs/occasional_papers/ 2010/RAND_OP323.pdf

- Kognit: http://www.kognit.dfki.de 


\subsection{Journals}

- $\mathrm{AI}$ in Medicine: http://www.aiimjournal.com

- Journal of Medical Imaging: http://www.spie.org

- Special Issue of ACM TMIS: http://www.dl.acm.org/ citation.cfm?id=2555810

- Special Issue of JAIR (AAAI FS workshop): http:// www.adventiumlabs.com/HIAI14

- Journal of the American Medical Informatics Association: http://www.jamia.oxfordjournals.org

\subsection{Conferences}

- Computer-Based Medical Systems (CBMS): http:// www.sites.ieee.org/cbms/

- Artificial Intelligence in Medicine (AIME): http:// www.aime15.aimedicine.info

- Health 2.0 Europe: http://www.health2con.com/events/ conferences/spring-fling-barcelona-2015/

- Digital Health Days: http://www.digitalhealthdays.se

- Health 2.0 Fall Conference 2015: http://www.health2 con.com/events/conferences/health-2-0-fall-2015/

- eHealth conference: http://www.ehealth.gvg.org

\subsection{Open Software and Datasets}

- i2b2: https://www.i2b2.org/resrcs/tools.html

- i2b2 NLP challenge: https://www.i2b2.org/NLP/Heart Disease/
- The tranSMART platform: http://www.transmartfoun dation.org/developers/

- Knowledge Management for Medical Care: http:// www.openclinical.org

- Modelling Wiki: https://www.moki.fbk.eu

- Workflow Automation: http://www.camunda.org

- Medical Guidelines: http://www.acr.org/Quality-Safety/ Standards-Guidelines

- CRISTAL-ISE Modelling Tool: http://www.cristal-ise. org

- Linking Open Drug Data (LODD): http://www.w3.org/ wiki/HCLSIG/LODD

\subsection{Commercial Hardware and Software}

- Scanadu: https://www.scanadu.com/scout/

- Spire: https://www.spire.io

- Omsignal: http://www.omsignal.com

- OpenHRE Toolkit: http://www.browsersoft.com/open hre.html

- Rockheatlh Resources: http://www.rockhealth.com/ resources/rock-reports/ 